\title{
Vascular Flora of the Chouteau Wildlife Management Area Wagoner County, Oklahoma
}

\author{
Bruce W. Hoagland \\ Oklahoma Biological Survey \\ and Department of Geography \\ University of Oklahoma \\ Norman, OK 73019 \\ e-mail: bhoagland@ou.edu
}

\author{
Forrest Johnson (deceased) \\ Oklahoma Biological Survey \\ University of Oklahoma \\ Norman, OK 73019
}

This article reports the results of a vascular plant inventory of the Chouteau Wildlife Management Area in eastern Oklahoma. One hundred eighty-one species of vascular plants were collected from 144 genera and 63 families. The families with the greatest number of species were the Asteraceae (25), Poaceae (22), and Fabaceae (18). Fifty-seven species were annuals, four biennials, and 120 were perennials. Thirty-nine woody plant species were present. Twenty-one species exotic to North America were collected representing $\mathbf{1 1 . 6 \%}$ of the flora. Azolla caroliniana was the only species tracked by the Oklahoma Natural Heritage Inventory found. This study reports 148 species previously not documented in Wagoner County.

\section{INTRODUCTION}

The objectives of this study were twofold: to fill a gap in floristic data for eastern Oklahoma and provide resource managers at the Chouteau Wildlife Management Area (CHWMA) with a comprehensive species list. Prior to 1996, when collecting began for this study, 198 specific and infraspecific taxa were reported from Wagoner County (Hoagland 2004). The first collections made in Wagoner County were by Robert Bebb, namesake of the University of Oklahoma Herbarium, in 1903 (Hoagland 2004). No additional collections were recorded until 1913, when G. W. Stevens visited the county. The peak collecting year in Wagoner County was 1939 (51 specimens), with work completed by R. Bebb (Hoagland et al. 2004).

\section{STUDY AREA}

The CHWMA is located on U.S. Army Corp of Engineers land in Wagoner County (Figure 1) and has been managed by the Oklahoma Department of Wildlife Conservation since 1973. It encompasses 402 hectares, and elevation ranges from $167 \mathrm{~m}$ to $158 \mathrm{~m}$. Latitudinal extent ranges

Hoagland, B.W. and Johnson, F.L.

https://doi.org/10.22488/okstate.17.100030 from $35.860 \mathrm{~N}$ to $35.85 \mathrm{o} \mathrm{N}$ and longitudinal extent from $95.34 \mathrm{o} \mathrm{W}$ to $95.37 \mathrm{o} \mathrm{W}$. The CHWMA is located within the subtropical humid (Cf) climate zone (Trewartha 1968). Summers are warm (mean July temperature $=27.7 \mathrm{o} C$ ) and humid, whereas winters are relatively short and mild (mean January temperature $=2.90 \mathrm{C}$ ). Mean annual precipitation is $114.5 \mathrm{~cm}$, with periodic severe droughts (Oklahoma Climatological Survey 2004).

Physiographically, the study area is located in the Osage Plains section of the Central Lowlands province (Hunt 1974) and within the Claremore Cuesta Plains province of Oklahoma (Curtis and Ham 1979). The surface geology is primarily Quaternary silt, sand, and clays deposited along the Verdigris River (Branson and Johnson 1979). The primary soil association at CHWMA is the Sage-Radley, which is composed of deep, level to gently sloping, poorly drained soils (Polone 1976). The potential natural vegetation type at CHWMA is the bottomland Forest type (Duck and Fletcher 1943). 


\section{METHODS}

Three collection sites were established at CHWMA for intensive floristic sampling. Sites were selected following a review of US Geological Survey 1:24,000 topographic maps and field reconnaissance. The predominant vegetation associations at these sites were classified according to Hoagland (2000). Collections also were made randomly throughout the site. Collections were made on a monthly basis from March through October 1996. Vouchers for species exotic to North America were made from naturalized populations only, thus excluding cultivated and ornamental plants. Specimens were processed at the Robert Bebb Herbarium of the University of Oklahoma (OKL) following standard herbarium techniques. Specimens were identified using Waterfall (1969) and Diggs et al. (1999). Origin (whether native or introduced to North America) was determined using Taylor and Taylor (1991) and United States Department of Agriculture-Natural Resources Conservation Service (USDA-NRCS 2004). Nomenclature follows USDA-NRCS (2004). Voucher specimens were deposited at OKL.

\section{RESULTS AND DISCUSSION}

A total of 181 vascular plants in 144 genera and 63 families were collected (Table 1). Among the angiosperms, 43 were monocots and 142 were dicots. The most species were collected from the families Poaceae (22), Asteraceae (25), Fabaceae (18). The genera Polygonum (6) and Carex (5) had the most species. Fifty-seven species were annual, four biennials, and 119 perennial. Thirty-nine woody plant species were present.

Twenty-one exotic species were collected, representing $11.6 \%$ of the flora. The numbers of exotic species were greater in the families Poaceae (6) and Fabaceae (7). These numbers are comparable to recent floristic inventories from other areas in Oklahoma. For example, a flora of the Chickasaw National Recreation Area reported 12\% exotic species (Hoagland and Johnson 2001),
9\% at Oologah Wildlife Management Area (Hoagland and Wallick 2003), 15\% at Keystone Wildlife Management Area (Hoagland and Buthod 2003), and 11\% for an inventory of Tillman County (Hoagland et al. 2004). However, the percentage was lower, 6.6\%, at Red Slough and Grassy Slough in southeastern Oklahoma (Hoagland and Johnson 2004). However, these studies report a higher number of exotic species in the Asteraceae. In addition, CHWMA is the first reported location for Alternanthera philoxeroides in Oklahoma, a noxious weed of the southeastern United States (Hoagland and McCarty 1998). Azolla caroliniana (G5S2) was the only species tracked by the Oklahoma Natural Heritage Inventory found at CHWMA. Species are ranked according to level of imperilment at the state $(S)$ and global $(G)$ levels on a scale of $1 \cdot 5 ; 1$ representing a species that is imperiled and 5 representing one that is secure (Groves et al. 1995).

As a result of this study, 313 species are now known to occur in Wagoner County. Of the 181 species reported in this study, 33 had been previously collected in the county. There were 165 species reported in the Atlas of the Flora of Oklahoma database that were not reported in this study (Hoagland 2004). This study documented 148 species not previously reported from Wagoner County. The three collection sites occurred within four vegetation associations. A brief description of each follows:

\section{Aquatic and wetland vegetation}

Several aquatic and wetland vegetation types were present at CHWMA. All intergraded with one another, making clear delineations difficult. The predominant emergent wetland vegetation types were Jussiaea peploides - Polygonum bydropiperoides herbaceous association, Nelumbo lutea herbaceous association, and Juncus effusus herbaceous association. Cephalantbus occidentalis shrubland association was the predominant woody wetland vegetation type. Associated 
species included Hibiscus laevis, Justicia americana, Potamogeton nodosus, Polygonum lapathifolium, P. pensylvanicum, Salix nigra, and Typha domingensis.

Azolla caroliniana, a species tracked by the Oklahoma Natural Heritage Inventory (2004), was found in this habitat type.

\section{Quercus palustris - Carya illinoensis/Ilex decidua forest association}

This association was the predominant forest type at CHWMA. However, all stands were immature second growth. Associate species included Amorpha fruticosa, Ampelopsis cordata, Arundinaria gigantea, Fraxinus pennsylvanica, Gleditsia triacanthos, Passiflora lutea and Ulmus rubra. On natural levies along the Verdigris River this association intergraded with the Acer saccharinum - Acer negundo forest association.

\section{Disturbed areas and old-field vegetation}

This designation included areas which have been or are currently in cultivation, roadsides and areas visited by CHWMA visitors, and other areas exhibiting signs of physical disruption. Common plants in disturbed areas and old fields included: Ambrosia trifida, Geranium carolinianum, Melilotus officinalis, Oenothera biennis, Solanum carolinense, Sorghum halepense, and Trifolium dubium.

\section{ACKNOWLEDGMENTS}

This project was funded by a grant from the Oklahoma Department of Wildlife Conservation.

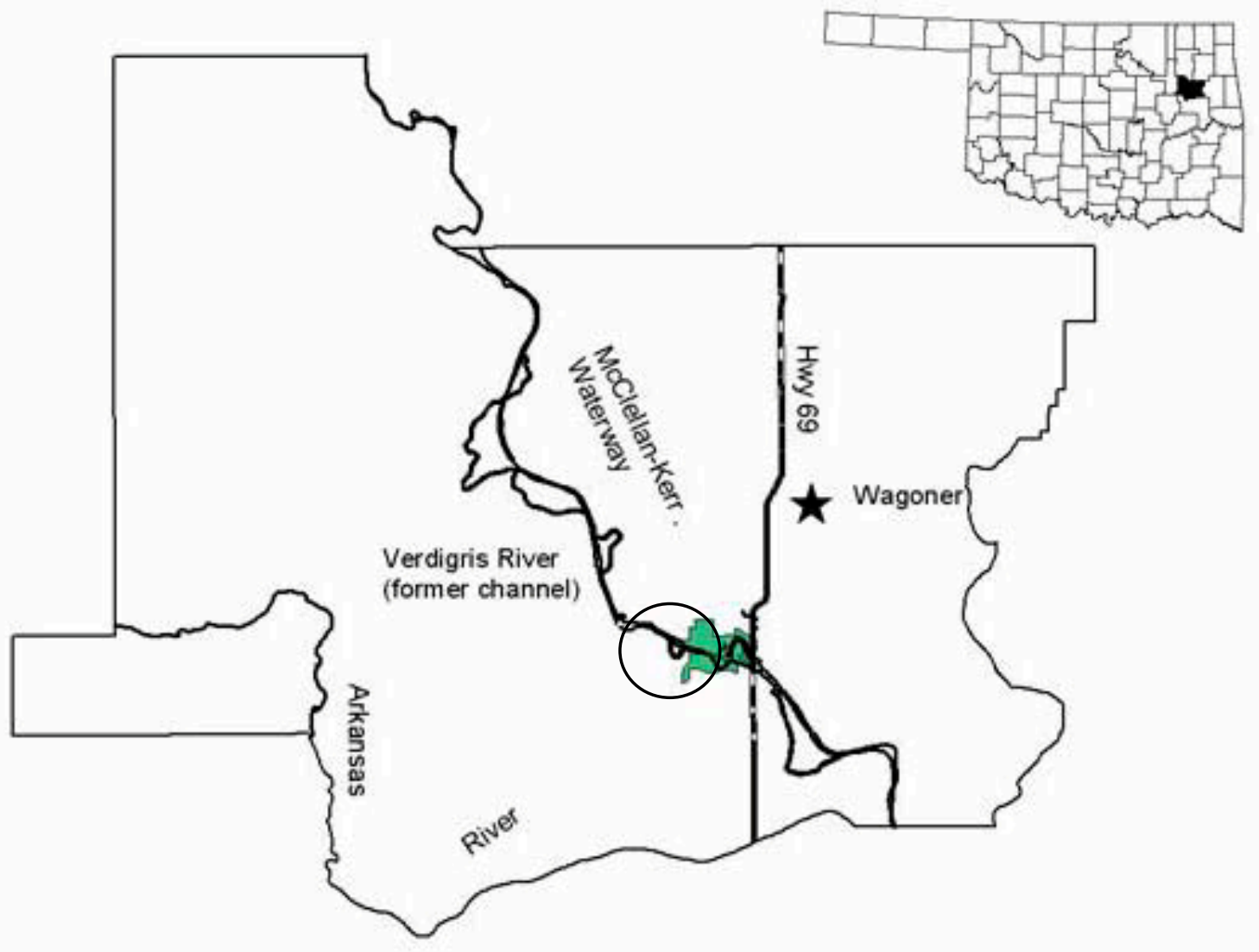

Figure Location of Chouteau Wildlife Management Area, Wagoner County, Oklahoma, site of the floristic collection.

Hoagland, B.W. and Johnson, F.L. 


\section{Annotated Species List for the Chouteau Wildlife Management Area}

The first entry is life history ( $\mathrm{A}=$ annual, $\mathrm{B}=$ biennial, $\mathrm{P}=$ perennial); followed by abundance $(1=$ least $5=$ dominant or codominant, Palmer et al. 1995); species not native to North America designated with an asterisk $\left(^{*}\right)$; habitat $(\mathrm{AQ}=$ Aquatic and wetland vegetation, $\mathrm{BF}=$ Quercus palustris $\bullet$ Carya illinoensis/Ilex decidua forest association, and DAOF=disturbed area/old-field); and collection number. Voucher specimens were deposited at the Robert Bebb Herbarium at the University of Oklahoma (OKL).

\section{PTERIDOPHYTA}

\section{Azollaceae}

Azolla caroliniana Willd. (mosquito fern) A; 2; AQ; CH096

MAGNOLIOPHYTA

MAGNOLIOPSIDA

\section{Acanthaceae}

Justicia americana (L.) Vahl (water willow) P; 2; AQ; CH037

Ruellia strepens $\mathrm{L}$. (wild petunia)

P; 2; BF; CH0173

Aceraceae

Acer negundo L. (boxelder)

P; 3; BF; CH079

A. saccharinum $\mathrm{L}$. (silver maple)

P; 2; BF; CH078

Amaranthaceae

Alternanthera philoxeroides (Mart.) Griseb.*

(alligator weed)

P; 3; AQ; CH094

Amaranthus palmeri $\mathrm{S}$. Wats.

(Palmer's pigweed)

A; 2; DAOF; CH0144

Apiaceae

Limnosciadium pinnatum (DC.) Mathias \&

Constance (tansy dog shade)

A; 3; AQ; CH065

Ptilimnium capillaceum (Michx.) Raf.

(threadleaf mockbishopweed)

A; 2; DAOF; CH0134

Sanicula canadensis L. (snakeroot)

B; 2; BF; CH0143

Torilis arvensis (Huds.) Link.*

(hedge parsley)

A; 2; DAOF; CH063

Apocynaceae

Apocynum cannabinum L. (Indian hemp)

P; 3; DAOF; CH085

Aquifoliaceae

Ilex decidua Walt. (deciduous holly)
P; 3; BF; CH0114

\section{Aristolochiaceae}

Aristolochia tomentosa Sims (wooly pipe vine)

P; 2; BF; CH0101

\section{Asclepiadaceae}

Asclepias incarnata L. (swamp milkweed)

P; 2; AQ; CH0160

A. viridis Walt. (green milkweed)

P; 2; DAOF; CH072

Asteraceae

Ageratina altissima (L.) King \& H.E. Robins.

(white snakeroot)

P; 2; DAOF; CH0194

Ambrosia artemisiifolia L. (common ragweed)

A; 3; DAOF; CH0174

A. trifida L. (giant ragweed)

A; 4; DAOF; CH0157

Bidens aristosa (Michx.) Britt.

(bearded beggarticks)

A; 2; AQ; CH0206

Boltonia asteroides (L.) L'Her. var. latisquamata

(Gray) Cronq. (white doll's daisy)

P; 2; AQ; CH0208

Cirsium altissimum (L.) Hill (tall thistle)

B; 2; DAOF; CH0185

Conoclinium coelestinum (L.) DC.

(blue mistflower)

P; 2; AQ; CH0199

Conyza canadensis (L.) Cronq. (horseweed)

A; 3; DAOF; CH0162

Coreopsis tinctoria Nutt. (plains coreopsis)

A; 3; DAOF; CH0123

Dracopis amplexicaulis (Vahl.) Cass.

(clasping coneflower)

A; 4; AQ, DAOF; CH073

Eclipta prostrata (L.) L. (yerba de tajo)

P; 3; AQ; CH0108

Elephantopus carolinianus Raeusch.

(elephant's foot)

P; 2; BF; CH0150 
Erigeron strigosus Muhl. ex Willd.

(daisy fleabane)

B; 2; DAOF; CH090

Grindelia papposa Nesom \& Suh (goldenweed)

A; 2; DAOF; CH0111

Helianthus annuus L. (common sunflower)

A; 2; DAOF; CH0164

Iva annua $\mathrm{L}$. (marsh elder)

A; 3; DAOF; CH0158

Lactuca serriola L.* (prickly lettuce)

A; 2; DAOF; CH0145

Pyrrhopappus multicaulis (D. Don) DC.

(Geiser's false dandelion)

P; 2; DAOF; CH060

Solidago canadensis L. (Canada goldenrod)

P; 2; DAOF; CH0197

Symphyotrichum ericoides (L.) Nesom

(white heath aster)

P; 2; DAOF; CH0189

S. ontarione (Wieg.) Nesom (bottomland aster)

P; 2; DAOF; CH0200

S. subulatum (Michx.) Nesom (eastern

saltmarsh aster)

A; 4; AQ; CH0165

Verbesina virginica $\mathrm{L}$. (frostweed)

P; 2; BF; CH0184

Vernonia baldwinii Torr. (western ironweed)

P; 2; DAOF; CH0163

Xanthium strumarium L. (cocklebur)

A; 2; AQ; CH0209

\section{Balsaminaceae}

Impatiens capensis Meerb. (jewelweed)

A; 2; BF; CH0109

Bignoniaceae

Campsis radicans (L.) Seem. ex Bureau

(trumpetvine)

P; 2; BF; CH083

Brassicaceae

Lepidium densiflorum Schrad. (peppergrass)

A; 2; DAOF; CH051

Rorippa palustris (L.) Bess (bog yellow cress)

A; 2; AQ; CH088

Thlaspi arvense L.* (field pennycress)

A; 1; DAOF; CH053

Campanulaceae

Triodanis perfoliata (L.) Nieuw.

(clasping Venus' looking glass)

A; 2; DAOF; CH082

\section{Caprifoliaceae}

Sambucus nigra L. ssp. canadensis (L.) R. Bolli (elderberry) P; 2; BF; CH084

Viburnum rufidulum Raf. (rusty blackhaw)

P; 2; BF; CH074

Celastraceae

Euonymus atropurpurea Jacq. (wahoo)

P; 2; BF; CH0187

Chenopodiaceae

Chenopodium standleyanum Aellen

(Standley's goosefoot)

A; 3; DAOF; CH0159

\section{Convolvulaceae}

Ipomoea lacunosa L. (white morning glory)

A; 2; DAOF; CH0203

I. pandurata (L.) G.F.W. Mey.

(bigroot morning glory)

P; 3; DAOF; CH0129

\section{Cornaceae}

Cornus drummondii C.A. Mey.

(rough leaved dogwood)

P; 3; DAOF; CH069

Crassulaceae

Penthorum sedoides L. (ditch stonecrop)

P; 3; AQ; CH0176

Ebenaceae

Diospyros virginiana $\mathrm{L}$. (persimmon)

P; 2; DAOF; CH025

Euphorbiaceae

Chamaesyce maculata (L.) Small

(spotted spurge)

A; 3; DAOF; CH0151

Euphorbia spathulata Lam. (warty spurge)

A; 2; DAOF; CH049

Fabaceae

Amorpha fruticosa L. (false indigo)

P; 2; AQ; CH052

Cercis canadensis $\mathrm{L}$. (redbud)

P; 3; BF; CH0170

Desmanthus illinoensis (Michx.) MacM. ex B.L.

Robins. \& Fern. (bundleflower)

P; 2; DAOF; CH0125

Desmodium paniculatum (L.) DC.

(panicled tickclover)

P; 3; BF; CH0106

Gleditsia triacanthos L. (honey locust)

P; 3; BF; CH044

Gymnocladus dioicus (L.) K. Koch.

Hoagland, B.W. and Johnson, F.L. 
(Kentucky coffee tree)

P; 2; BF; CH092

Lathyrus pusillus Ell. (low peavine)

A; 2; DAOF; CH0O2

Lespedera cuneata (Dum.-Cours.) G. Don*

(sericea lespedeza)

P; 2; DAOF; CH0167

Melilotus alba Medikus* (white sweet clover) A; 2; DAOF; $\mathrm{CH} 071$

M. officinalis (L.) Lam.* (yellow sweet clover) A; 3; DAOF; CH041

Senna marilandica (L.) Link (wild senna)

P; 2; BF; CH0124

Sesbania herbacea (P. Mill.) McVaugh (bequilla)

A; 5; AQ; CH0166

Strophostyles helvola (L.) Ell.

(fuzzy trailing bean)

A; 2; DAOF; CH0191

Trifolium arvense L.* (rabbit foot clover)

A; 2; DAOF; $\mathrm{CH} 040$

T. dubium Sibthrop* (small hop clover)

A; 2; DAOF; $\mathrm{CH} 026$

T. pratense L.* (red clover)

P; 2; DAOF; CH0140

Vicia caroliniana Walt. (pole vetch)

P; 3; DAOF; CH0128

$V$. villosa Roth* (hairy vetch)

A; 3; DAOF; CH035

Fagaceae

Quercus macrocarpa Michx. (bur oak)

P; 2; BF; CH0135

Q. palustris Muenchh. (pin oak)

P; 3; BF; CH034

Q. velutina Lam. (black oak)

P; 2; BF; CH042

Geraniaceae

Geranium carolinianum L. (Carolina cranesbill)

A; 2; DAOF; $\mathrm{CH} 027$

Juglandaceae

Carya illinoensis (Wangenh.) K. Koch (Pecan)

P; 2; BF; CH087

Lamiaceae

Prunella vulgaris L. (Common self heal)

P; 2; BF; CH020

Lauraceae

Sassafras albidum (Nutt.) Nees (sassafras)

P; 2; BF; CH0120

\section{Lythraceae}

Ammannia coccinea Rottb. (redstem loosestrife)

A; 2; AQ; CH0141

Lythrum alatum Pursh (winged loosestrife)

P; 2; AQ; CH0121

Malvaceae

Hibiscus laevis All. (halberd leaved rose

mallow) P; 2; AQ; CH0153

Sida spinosa L. (prickly sida)

A; 1; DAOF; CH0152

Menispermaceae

Calycocarpum lyonii (Pursh) Gray (cupseed)

$\mathrm{P} ; 2$; BF; CH093

Cocculus carolinus (L.) DC. (Carolina snailseed)

P; 2; BF; CH0103

Moraceae

Morus rubra L. (red mulberry)

P; 2; BF; CH0180

Nelumbonaceae

Nelumbo lutea Willd. (Lotus)

P; 2; AQ; CH0179

Oleaceae

Fraxinus pennsylvanica Marsh. (green ash)

P; 3; BF; CH043

\section{Onagraceae}

Ludwigia palustris (L.) Ell. (marsh seedbox)

P; 4; AQ; CH055

L. repens Forst. (water primrose)

P; 2; AQ; CH0131

Oenothera biennis L.

(common evening primrose)

B; 3; DAOF; CH0161

O. laciniata Hill (cutleaf evening primrose)

A; 2; DAOF; CH061

Oxalidaceae

Oxalis stricta L. (yellow wood sorrel)

P; 2; DAOF; CH081

Passifloraceae

Passiflora lutea L. (yellow passionflower)

P; 2; BF; CH058

Phytolaccaceae

Phytolacca americana L. (pokeweed)

P; 2; DAOF; CH0116

Polygonaceae

Polygonum hydropiper L.* (water pepper)

A; 2; AQ; CH0115

P. bydropiperoides Michx.* (mild water pepper)

P; 4; AQ; CH0113 
P. lapathifolium L. (pale smartweed)

A; 3; AQ; CH0190

P. pensylvanicum L. (Pennsylvania smartweed)

A; 2; AQ; CH0204

P. ramosissimum Michx. (knotweed)

A; 2; AQ; $\mathrm{CH} 014$

P. scandens $\mathrm{L}$. (false buckwheat)

P; 2; AQ; CH0193

Rumex altissimus Wood (pale dock)

P; 2; DAOF; CH089

R. crispus L.* (curly dock)

P; 3; DAOF; CH091

R. verticillatus $\mathrm{L}$. (Water dock)

P; 2; DAOF; CH07

Ranunculaceae

Clematis pitcheri Torr. \& Gray (Pitcher's

clematis) P; 2; BF; CH046

Ranunculus sceleratus L. (cursed buttercup)

A; 2; AQ; CH031

\section{Rosaceae}

Crataegus viridis L. (green hawthorn)

P; 3; BF; CHO6

Geum canadense Jacq. (white avens)

P; 2; BF; CH0112

Rosa multiflora Thunb. ex Murr.*

(Japanese rose)

P; 2; DAOF; CH033

R. setigera Michx. (climbing prairie rose)

P; 2; DAOF; CH056

Rubus trivialis Michx. (southern blackberry)

P; 3; BF; CH0105

Rubiaceae

Cephalanthus occidentalis L. (buttonbush)

P; 2; AQ; CH0138

Galium aparine L. (catchweed bedstraw)

A; 2; BF; CH036

Spermacoce glabra Michx. (smooth buttonweed)

P; 2; AQ; CH0155

Salicaceae

Salix nigra Marsh. (black willow)

P; 2; AQ; CH0192

Sapindaceae

Sapindus saponaria L. var. drummondii

(Hook. \& Arn.) L. Benson (soapberry)

P; 2; BF; CH077

\section{Sapotaceae}

Sideroxylon lanuginosum Michx. (chittamwood) P; 2; BF; CH0110

\section{Scrophulariaceae}

Lindernia dubia (L.) Pennell (false pimpernel)

A; 2; AQ; CH0136

Penstemon digitalis Nutt. ex Sims

(smooth penstemon)

P; 2; DAOF; CH045

Veronica peregrina L. (purslane speedwell)

A; 2; DAOF; CH024

Solanaceae

Physalis angulata L. (cutleaf ground cherry)

A; 2; DAOF; CH015

Solanum carolinense $\mathrm{L}$. (Carolina horsenettle)

P; 2; DAOF; CH062

Ulmaceae

Celtis laevigata Willd. (sugarberry)

P; 4; BF; CH01

Ulmus alata Michx. (winged elm)

P; 3; BF; CH032

U. rubra Muhl. (slippery elm)

P; 4; BF; CH038

Urticaceae

Boebmeria cylindrica (L.) Sw. (false nettle)

P; 2; BF; CH0175

Valerianaceae

Valerianella radiata (L.) Dufr.

(common beaked cornsalad)

A; 2; AQ; CH08

Verbenaceae

Phyla lanceolata (Michx.) Greene

(northern fogfruit)

P; 2; AQ; CH0139

Viscaceae

Phoradendron leucarpum (Raf.) Reveal \& M.C.

Johnston (eastern mistletoe)

P; 2; BF; CH086

Vitaceae

Ampelopsis arborea (L.) Koehne (peppervine)

P; 2; BF; CH0100

A. cordata Michx. (racoon grape)

P; 2; BF; CH0147

Parthenocissus quinquefolia (L.) Planch.

(Virginia creeper)

P; 3; BF; CH098

Vitis aestivalis Michx. (pigeon grape)

P; 3; BF; CH0102

$V$. cinerea (Engelm.) Millard (sweet grape)

P; 2; BF; CH0107

Hoagland, B.W. and Johnson, F.L. 


\section{LILIOPSIDA}

\section{Alismataceae}

Echinodorus cordifolius (L.) Griesb.

(creeping burhead)

P; 2; AQ; CH0177

Sagittaria latifolia Willd. (duck potato)

P; 2; AQ; CH0186

\section{Araceae}

Arisaema dracontium (L.) Schott (green dragon) P; 2; BF; CH0114

\section{Cyperaceae}

Carex crus-corvi Shuttlw. ex Kunze

(ravenfoot sedge)

P; 2; AQ; CH070

C. granularis Muhl. ex Willd. var. haleana

(Olney) Porter (Limestone meadow

sedge)

P; 2 BF; CH0032

C. byalinolepis Steudel (shoreline sedge)

P; 2; AQ; CH0089

C. tribuloides Wahlenberg (blunt broom sedge)

P; 2 BF; CH0103

C. vulpinoidea Michx. (fox sedge)

P; 2 BF; CH0230

Cyperus pseudovegetus Stued.

(marsh flatsedge)

P; 2; AQ; CH0114

C. strigosus $\mathrm{L}$. (strawcolored flatsedge)

P; 2; AQ; CH097

Eleocharis compressa Sullivant

(flatstem spikesedge)

P; 4; AQ; CH052

E. obtusa (Willd.) J.A. Schultes

(blunt spikesedge)

P; 2; AQ; CH0039

\section{Iridaceae}

Sisyrinchium angustifolium P. Mill.

(blue eyed grass)

P; 2; DAOF; CH0029

Juncaceae

Juncus acuminatus Michx. (tapertip rush)

P; 2; AQ; CH063

J. effusus L. (soft rush)

P; 2; AQ; CH024

J. interior Wieg. (inland rush)

P; 2; AQ; CH041

Liliaceae

Allium canadense L. (wild onion)

$$
\text { P; 2; DAOF; CH030 }
$$

Poaceae

Agrostis byemalis (Walt.) B. S. P. (ticklegrass)

P; 2; AQ; CH0017

Alopecurus carolinianus Walt. (Carolina foxtail)

A; 2; AQ; CH0019

Andropogon glomeratus (Walt.) B. S. P.

(broomsedge)

P; 3; DAOF; CH0182

Arundinaria gigantea (Walt.) Mulh. (giant cane)

P; 2; BF; CH076

Bromus japonicus Thunb. ex Murr*.

(Japanese brome)

P; 3; DAOF; CH047

Digitaria sanguinalis (L.) Scop. (hairy crabgrass)

A; 3; DAOF; CH0169

Echinochloa colona (L.) Link* (barnyard grass)

A; 2; AQ; CH0205

E. crus-galli (L.) Beauv.* (barnyard grass)

A; 3; AQ; CH0104

E. muricata (Beauv.) Fern.* (barnyard grass)

A; 2; AQ; CH0130

Elymus virginicus $\mathrm{L}$. (Virginia wild rye)

P; 2; BF; CH075

Eragrostis spectabilis (Pursh.) Steud.

(purple lovegrass)

P; 2; BF; CH0196

Hordeum pusillum Nutt. (little barley)

A; 3; DAOF; CH050

Leersia oryzoides (L.) Sw. (rice cutgrass)

P; 2; AQ; CH0181

Leptochloa panicea (Retz.) Ohwi ssp. brachiata

(Steudl.) N. Snow (red sprangletop)

A; 2; AQ; CH0201

Lolium perenne L.* (perennial ryegrass)

P; 2; DAOF; CH048

Panicum dichotomiflorum Michx. (fall panicum)

A; 2; BF; CH0198

Paspalum pubiflorum Rupr. ex Fourn.

(hairyseed paspalum)

P; 2; DAOF; CH0202

Setaria parviflora (Poir.) Kerguélen.

(knotroot bristlegrass)

P; 2; DAOF; CH0207

S. viridis (L.) Beauv.* (green foxtail)

A; 2; DAOF; CH0127

Sorghum halepense (L.) Pers.*

(Johnson grass) 
P; 3; DAOF; CH021

Sphenopholis obtusata (Michx) Scribn.

(wedgegrass)

P; 2; AQ; CH010

Tridens flavus (L.) A.S. Hitchc. (redtop)

P; 3; DAOF; CH0183

Potamogetonaceae

Potamogeton nodosus Poir.

(long leaved pondweed)
P; 2; AQ; CH095

Smilacaceae

Smilax bona-nox L. (greenbriar)

P; 2; BF; CH097

$S$. glauca Walt. (pale greenbriar)

P; 2; BF; CH0119

Typhaceae

Typha domingensis Pers. (southern cattail)

P; 2; AQ; CH0178

Table Summary of floristic collections at the Chouteau Wildlife Management Area, Wagoner County, Oklahoma. Table format follows Palmer et al. (1995).

\begin{tabular}{llll}
\hline Taxonomic Group & Species & Native spp. & Introduced spp. \\
Pteridophyta & 1 & 1 & 0 \\
Magnoliophyta & 137 & 122 & 15 \\
Magnoliopsida & 43 & 37 & 6 \\
Liliopsida & 181 & 160 & 21 \\
\hline Total & & & \\
\hline
\end{tabular}

\section{LITERATURE CITED}

Branson, C.C. and K.S. Johnson. 1979.

Generalized geologic map of Oklahoma.

Page 4 in K.S. Johnson, C.C.

Branson, N.M. Curtis, W. E. Ham,

W.E. Harrison, M.V. Marcher, and

J.F. Roberts, editors, Geology and

Earth Resources of Oklahoma.

Oklahoma Geological Survey,

Norman.

Curtis, N.M. and W.E. Ham. 1979.

Geomorphic provinces of Oklahoma. Page
45 in K.S. Johnson, C.C. Branson, N.M. Curtis, W.E. Ham, W.E. Harrison, M.V. Marcher, and J.F. Roberts, editors, Geology and Earth Resources of Oklahoma. Oklahoma Geological Survey, Norman.

Diggs, G.M., B.L. Lipscomb, and R.J. O'Kennon. 1999. Shinners and Mabler's Illustrated Flora of North Central Texas. Botanical Research Institute of Texas and Austin College, Fort Worth.

Hoagland, B.W. and Johnson, F.L. 
Duck, L.G. and J.B. Fletcher. 1943. A game type map of Oklahoma. Oklahoma Department of Wildlife Conservation, Oklahoma City. Groves, C.R., M.L. Klein, and T.F. Breden. 1995. Natural Heritage Programs: public-private partnerships for biodiversity conservation. Wildlife Society Bulletin 23:784-790.

Hoagland, B.W. 2000. The vegetation of Oklahoma: a classification of landscape mapping and conservation planning. Southwest Naturalist 45:385-420.

Hoagland, B.W. 2004. Atlas of the flora of Oklahoma [online]. Available: www.biosurvey.ou.edu. (Accessed on 14 January 2004).

Hoagland, B.W. and A. Buthod. 2003. $V$ ascular flora of the Keystone Wildlife Management Area, Creek, Pawnee, and Osage counties, Oklahoma. Oklahoma Native Plant Record 3:23-37.

Hoagland, B.W., P. Crawford-Callahan, P. Crawford, and F.L. Johnson. 2004. V ascular Flora of Hackberry Flat, Frederick Lake, and Suttle Creek, Tillman County, Oklahoma. Sida 21:429-445.

Hoagland, B.W. and F.L. Johnson. 2001. Vascular flora of the Chickasaw National Recreation Area, Murray County, Oklahoma. Castanea 66:383-400.

Hoagland, B.W. and F.L. Johnson. 2004. The vascular flora of Red Slough and Grassy Slough Wildlife Management Areas, Gulf Coastal Plain, McCurtain County, Oklahoma. Castanea 69.

Hoagland, B. W. and N. A. McCarty. 1998. Alternanthera philoxeroides (Mart.) Griseb.(AMARANTHACEAE) new to Oklahoma. Castanea 63: 194.
Hoagland, B.W. and K. Wallick. 2003. Vascular flora of Oologah Wildlife Management Area, Nowata County, Oklahoma. Proceedings of the Oklahoma Academy 83:47-62.

Hunt, C.B. 1974. Natural Regions of the United States and Canada. W. H. Freeman, San Francisco.

Oklahoma Climatological Survey. 2004. Oklahoma Climatological Data [online]. Available: http://www.ocs.ou.edu/. (Accessed on 1 March 2004).

Oklahoma Natural Heritage Inventory. 2004. ONHI working list of rare Oklahoma plants [online]. Available: http://www.biosurvey. ou.edu/publicat.html. (Accessed on 1 March 2004).

Palmer, M.W., G.L. Wade, and P. Neal. 1995. Standards for the writing of floras. Bioscience 45:339-345.

Polone, D. J. 1976. Soil survey of $W$ agoner County, Oklahoma. United States Department of Agriculture, Washington D.C.

Taylor, R.J. and C.S. Taylor. 1991. An annotated list of the ferns, fern allies, gymnosperms, and flowering plants of Oklahoma. Southeastern Oklahoma State University, Durant.

Trewartha, G.T. 1968. An Introduction to Climate. McGraw-Hill, New York.

USDA-NRCS 2004. The PLANTS database [online].

Available: http://plants.usda.gov/pl ants. National Plant Data Center, Baton Rouge, LA. (Accessed on 14 January 2004).

Waterfall, U.T. 1969. Keys to the flora of Oklahoma. $4^{\text {th }}$ edition. Published by the author, Stillwater. 